\title{
Digitalisierte Arbeit und private Lebensführung
}

\author{
Martin Diewald, Eva Susanna Kunze und Björn Andernach
}

\section{Inhalt}

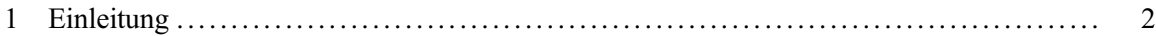

2 Auswirkungen von Beschäftigungsbedingungen auf das Privatleben: Stand der

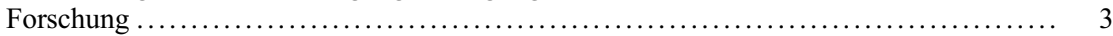

3 Digitalisierung und Beschäftigungsmerkmale: Was ändert sich für wen? . $\ldots \ldots \ldots \ldots \ldots \ldots$

4 Neue Aspekte digitalisierter Arbeit und vorliegende Untersuchungen zum

Zusammenhang zwischen digitalisierter Arbeit und Privatleben .................... 10

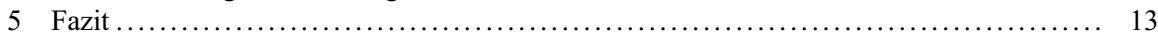

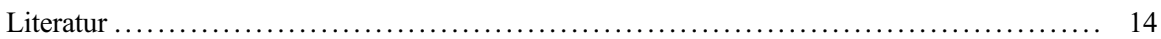

\section{Zusammenfassung}

Über die Auswirkungen digitalisierter Arbeitsumgebungen auf die private Lebensführung gibt es eher vage Vermutungen als belastungsfähige empirische Untersuchungen. Wir referieren deshalb hauptsächlich Untersuchungen zu allgemeinen Beschäftigungsbedingungen, die im Zuge von Digitalisierungsprozessen an Bedeutung zunehmen werden. Wir berücksichtigen, dass sich Digitalisierungsprozesse für verschiedene Beschäftigtengruppen durchaus unterschiedlich auswirken können. Darüber hinaus diskutieren wir spezifische Begleiterscheinungen von Digitalisierung auf der Ebene von Kommunikation und Interaktion, die von bisherigen Untersuchungen nicht hinreichend abgebildet werden konnten.

\section{Schlüsselwörter}

Work-Life-Konflikt · Freizeit · Familie · Mensch-Maschine-Interaktion · Crowdwork

\section{Diewald (ه) • E.S. Kunze • B. Andernach}

Fakultät für Soziologie, Arbeitsbereich Sozialstruktur und soziale Ungleichheit, Universität Bielefeld, Bielefeld, Deutschland

E-Mail: martin.diewald@uni-bielefeld.de; eva.kunze@uni-bielefeld.de; b.andernach@gmx.de 


\section{$1 \quad$ Einleitung}

In zahlreichen Veröffentlichungen zur Digitalisierung von Arbeit werden umfassende Vernetzung und Automatisierung, Dezentralisierung und der Umgang mit adaptiven, intelligenten und autonomen Systemen als Kern digitaler Arbeit genannt (z. B. Hermann et al. 2015; Krzywdzinski et al. 2015; Kagermann et al. 2013). Digitalisierung macht Informationen maschinenoperabel; die daraus resultierende Möglichkeit umfassender Datenerhebung und -auswertung verändert sowohl Struktur und Organisation von Arbeit als auch Anforderungen an die Beschäftigten (BMAS Grünbuch 2016b). Anwendungsgebiete digitalisierter Arbeit liegen neben Tätigkeiten im produzierenden Gewerbe (Stichworte: Smart Factory, Roboter) und in der Logistik-/Transportbranche (Smart Transportation Logistics) bspw. auch im Handwerk (Einrichten und Warten von Technologien für das Smart Home) sowie im tertiären Sektor (internetbasierte Dienstleistungen/Smart Services, bspw. im Bereich Handel und Beratung). Es handelt sich also um ein komplexes Bündel verschiedener Entwicklungen, die jedoch zusammengenommen die Arbeitswelt zunehmend verändern und weiter verändern werden. Dabei stellt sich die Frage, inwiefern dadurch nicht nur die Arbeit selbst, sondern auch die private Lebensführung verändert wird. Damit sind die außerhalb der Sphäre der Erwerbsarbeit angesiedelten Aktivitäten und Beziehungen gemeint, die insbesondere das Eingehen und die Stabilität familialer und nichtfamilialer Beziehungen, Freizeitaktivitäten sowie soziale und politische Partizipation umfassen. Eine umfassende, aussagekräftige Empirie zu Auswirkungen der Digitalisierung auf diese Facetten privater Lebensführung steht jedoch bisher kaum zur Verfügung. Auch wenn wir im Folgenden primär, wenn auch keineswegs ausschließlich, von soziologischen Beiträgen ausgehen, scheint uns auch insgesamt zu gelten, dass der bisherige Stand der Forschung sich weitgehend auf Vermutungen, die hierzu in Experteninterviews bzw. Meinungen und Einstellungen, die in Befragungen geäußert wurden, beschränkt. Die noch spärlichen empirischen Untersuchungen konzentrieren sich auf einzelne ausgewählte Digitalisierungsprozesse. Auch sind beim derzeitigen Entwicklungsstand der Digitalisierung Aussagen schwer möglich, inwiefern sie verschiedene Beschäftigtengruppen sehr unterschiedlich betreffen.

Bevor wir diesen noch sehr lückenhaften Stand der Forschung referieren (Abschn. 4), gehen wir deshalb einen zusätzlichen Weg. Gesellschaftlicher und technischer Wandel haben nämlich in den letzten Jahrzehnten nicht nur die Erwerbsarbeit selbst (Eichhorst et al. 2013), sondern auch die Zusammenhänge zwischen Berufs- und Privatleben merklich verändert. Diese Zusammenhänge sind gut erforscht. In diese Zeit, dem Ende der 1980er-Jahre, fällt bereits die flächendeckende Einführung von Computern als Vorläufer der derzeitigen Digitalisierung der Arbeit. Ausgehend von diesen Untersuchungen lässt sich bereits dreierlei vermuten. Erstens sind einfache utopische wie dystopische Zukunftsszenarien, so plausibel sie klingen mögen, kaum einmal eingetreten. Dies gilt sowohl für Hoffnungen einer umfassenden Befreiung von Arbeitszwängen (Gortz 1973) als auch spätere Befürchtungen einer weitgehenden Bedrohung der Bindungsfähigkeit (Sennett 1998). Zweitens hat sich gezeigt, dass verschiedene Beschäftigungsgruppen von Veränderungen sehr 
unterschiedlich betroffen sein können. Und drittens sind sowohl die Veränderungen in der Arbeitswelt selbst als auch die Auswirkungen auf die private Lebensführung in hohem Maße von betrieblichen und überbetrieblichen Gestaltungsmaßnahmen abhängig.

Mit der Digitalisierung der Arbeitswelt, in Deutschland auch unter dem fortschrittsbetonenden Begriff von „Industrie 4.0“ verhandelt (BMBF 2015), verbinden sich wieder ebensolche Hoffnungen und Befürchtungen. Die dabei im Fokus stehenden Beschäftigungsmerkmale - vor allem flexiblere und in den Anforderungen komplexere Arbeitsformen, zeitliche Entgrenzung der Arbeit und flexiblere Arbeitszeiten jenseits der Fünftagewoche zu Normalarbeitszeiten, unsicherere Arbeitsplätze sowie vermehrte Teamarbeit und mehr Entscheidungsnotwendigkeiten - stehen auch bei der voranschreitenden Digitalisierung mit im Vordergrund der Debatte (Abschn. 3). Dies ermöglicht uns, aus bisherigen Studien, vermittelt über diese Beschäftigungsmerkmale, Rückschlüsse auf erwartbare Auswirkungen der derzeitigen Digitalisierung in verschiedenen Beschäftigtengruppen zu ziehen, die mit den noch wenigen aktuellen Untersuchungen zu Auswirkungen der Digitalisierung auf das Privatleben noch nicht möglich sind (Abschn. 2). Abschließend fassen wir zusammen, welche Argumente und Befunde es dafür gibt, dass Digitalisierung im Vergleich zur dritten industriellen Revolution die Auswirkungen des Erwerbslebens auf das Privatleben neu gestaltet oder nur bisherige Entwicklungsprozesse fortführt und verstärkt (Abschn. 4).

\section{Auswirkungen von Beschäftigungsbedingungen auf das Privatleben: Stand der Forschung}

Im Folgenden werden wir zunächst den Stand der Forschung hinsichtlich derjenigen Beschäftigungsmerkmale referieren, die in bisherigen Untersuchungen einen Einfluss auf das Privatleben von Beschäftigten gezeitigt haben. Hierbei gehen wir kurz auf theoretische Grundlagen wie Rollentheorie und das Job-Demands-Resources Modell ein, anschließend werden empirische Ergebnisse aus folgenden Feldern vorgestellt: Konflikte zwischen Arbeit und privater Lebensführung, Eingehen und die Stabilität von (familiären) Beziehungen, soziale Beziehungen und Netzwerke außerhalb der Familie und Freizeitaktivitäten.

\subsection{Theoretische Ansätze}

Für die Analyse von möglichen Auswirkungen des Berufs- auf das Privatleben hat sich in der einschlägigen Forschung vor allem ein rollentheoretischer Ansatz durchgesetzt (Greenhaus und Beutell 1985). Konflikte entstehen demnach dann, wenn Rollenanforderungen im Berufs- und Privatleben in mindestens einer Hinsicht inkompatibel sind. Dabei hat sich gezeigt, dass solche Konflikte vor allem durch bestimmte Beschäftigungsbedingungen hervorgehoben werden (Allen et al. 2000), die vor allem über Belastung oder zeitliche Inkompatibilitäten Konflikte hervorrufen (Carlson et al. 2000). Ebenfalls im Hinblick darauf, ob Partnerschafts- und Eltern- 
beziehungen eingegangen werden, und auch dafür, wie stabil Partnerschaften sind, spielen rollentheoretische Überlegungen eine wichtige Rolle. Dabei geht es hauptsächlich darum, ob berufliche Unsicherheit das Eingehen stabiler privater Verpflichtungen verhindert (Sennett 1998) bzw. inwiefern existierende Partnerschaften dadurch destabilisiert werden, dass private Rollenanforderungen aufgrund von beruflichen Anforderungen nicht erfüllt werden (Cooke und Gash 2010) und Belastungen die Partnerschaftsqualität beeinträchtigen (Diewald et al. 2013). Allerdings sind auch kompensatorische Zusammenhänge bekannt, wenn etwa berufliche Sozialintegration Defizite privater Sozialintegration ausgleichen soll (Diewald 2003).

In der einschlägigen Forschung wird insbesondere zwischen den mit einer bestimmten beruflichen Tätigkeit verbundenen Ressourcen und Belastungen unterschieden (Bakker und Demerouti 2007). Berufliche Ressourcen können Konflikte vermeiden helfen und das Eingehen und die Gestaltung von privaten Beziehungen erleichtern, etwa wenn Zeitsouveränität und Autonomie eine bessere Abstimmung zwischen beruflichen und privaten Anforderungen erlauben oder Geld und Status die Pflege von Beziehungen erleichtern und teurere Freizeitaktivitäten ermöglichen. Umgekehrt drohen Belastungen wie Überstunden oder psychischer Stress in das Privatleben hineingetragen zu werden bzw. dem Leben in privaten Beziehungen Zeit und Energie zu entziehen. Zusätzlich werden im Rahmen des „psychologischen Vertrags“ (Rousseau 1995) Erwartungsenttäuschungen und Verletzungen des Gerechtigkeitsempfindens als mögliche Quellen von Konflikten thematisiert, die auf das Eingehen und die Gestaltung privater Beziehungen einen zusätzlichen negativen Einfluss haben können.

Während diese Ansätze eine individuenbezogene Sicht auf den Zusammenhang zwischen Berufs- und Privatleben haben, wird vor dem Hintergrund von international und zwischenbetrieblich vergleichenden Untersuchungen deutlich, dass Konflikte und Unvereinbarkeiten auch stark durch die institutionelle Ausgestaltung von Arbeitsmarkt- und Familienpolitik durch Politik und Arbeitgeber beeinflusst werden. Maßnahmen und Praktiken halten häufig noch nicht mit veränderten Lebensentwürfen und damit auch Anforderungen an die Arbeit Schritt oder bedienen eher die Flexibilitätsbedürfnisse der Arbeitgeber als die der Arbeitnehmer (Hobson 2014). Es gibt allerdings ein hohes Maß an Gestaltbarkeit - und auch faktischer Heterogenität - in der Ausformung von Arbeitsplatzmerkmalen und ihrer Bedeutung für das Privatleben (Moen et al. 2016).

\subsection{Empirische Ergebnisse}

\subsubsection{Wahrgenommene Konflikte zwischen Berufs- und Privatleben}

Die im Hinblick auf die Wirkung von Beschäftigungsbedingungen auf das Privatleben umfangreichste und detaillierteste Forschung richtet sich auf wahrgenommene Konflikte zwischen Berufs- und Privatleben (work-family conflict). Generell hat sich dabei gezeigt, dass Ressourcen im Vergleich zu Belastungen eine vergleichsweise geringere Bedeutung haben. Berufliche Autonomie wird überwiegend als Ressource 
identifiziert, die Konflikte vermeiden hilft (Voydanoff 2004; Grzywacz und Butler 2005; Schieman et al. 2009). Jedoch kann mit viel Verantwortung für andere und auch Selbstverantwortung in der Berufsausübung die Gefahr von Überlastung, Selbstausbeutung und eines Verlusts der Grenzen zwischen Berufs- und Privatleben einhergehen (Schieman et al. 2009; Böhm und Diewald 2012). Noch uneinheitlicher sind die Auswirkungen einer flexiblen Ausgestaltung von Arbeitszeiten. Offensichtlich kommt es hier entscheidend darauf an, wessen Flexibilitätsinteressen konkret im Vordergrund stehen: die des Arbeitgebers oder die der Beschäftigten. Je nachdem sind positive oder negative Folgen dokumentiert (z. B. Schier und Jurczyk 2007; Hill et al. 2010).

Weitgehend übereinstimmend in Studien für Deutschland und für andere Länder erhöhen sich Konflikte zwischen dem Berufs- und Privatleben vor allem bei folgenden Belastungen: (1) zuvorderst psychische Belastungen durch lang anhaltenden Arbeitsdruck, hohe Arbeitsverdichtung und komplexe Abstimmungsprozesse; sowie (2) lange Arbeitszeiten, Arbeit auch an Wochenenden und Feiertagen, Wechselschicht und die ständige Verfüg- und Erreichbarkeit durch moderne Informationsund Kommunikationssysteme (z. B. Böhm und Diewald 2012; Pausch et al. 2016; Gallie und Russell 2009; Schieman et al. 2009; Adkins und Premeaux 2012). Jobunsicherheit wirkt sich ebenfalls negativ aus, allerdings im Vergleich zu den anderen Belastungen in geringem Ausmaß (Beham und Drobnič 2010).

Kaum untersucht sind bisher Auswirkungen einer Verletzung des sogenannten psychologischen Vertrags, also das Enttäuschen von wechselseitigen, impliziten Erwartungen sowie die Bewertung der Fairness der Vertragsbeziehung. Eine neue Studie bestätigt für Deutschland, dass sowohl ein als unfair empfundenes Verhältnis von Geben und Nehmen in der Beschäftigungsbeziehung als auch speziell die Enttäuschung von Erwartungen hinsichtlich Autonomie und Flexibilität sowie eine angenehme Arbeitsatmosphäre Konflikte zwischen Beruf und Privatleben zusätzlich verschärfen (Reimann 2016).

Darüber hinaus hat sich auch die Organisationskultur, insbesondere in Form von Bereitschafts- und Flexibilitätserwartungen (Hobson und Fahlén 2009; für Deutschland: Drobnič und Guillén Rodríguez 2011; Pausch et al. 2016), als konfliktfördernd herausgestellt. Umgekehrt stellt die Unterstützung durch Vorgesetzte sowie durch Kolleginnen und Kollegen (z. B. Allen 2001; Goh et al. 2015; Hammer et al. 2009; für Deutschland: Reimann et al. 2016) eine wesentliche Ressource für die Vermeidung von Konflikten dar.

\subsubsection{Eingehen von Partnerschaft und Elternschaft}

Längsschnittliche Untersuchungen zum Eingehen von Partnerschaft und Elternschaft leiden generell darunter, dass in entsprechenden Lebenslauf- oder Panelstudien nur sehr wenige Informationen zu konkreten Beschäftigungsbedingungen enthalten sind. Diese beschränken sich im Wesentlichen auf die tatsächliche Arbeitszeit sowie die Unsicherheit von Beschäftigungsverhältnissen. Während diese Untersuchungen keinen Einfluss langer Arbeitszeiten ausweisen, kann Arbeitsplatzunsicherheit signifikant die Wahrscheinlichkeit verringern, dass Partnerschaften und eine Elternschaft eingegangen werden, Elternschaften allerdings eher bei höher qualifi- 
zierten Beschäftigten und abhängig von der Paarkonstellation (Kurz 2005; Schmitt 2008, 2012; Kreyenfeld 2010). Für prekäre Beschäftigung wie Leiharbeit, Minijobs und Zeitarbeit zeigen sich ebenfalls negative Auswirkungen ( $\mathrm{Laß} 2016$ ).

\subsubsection{Stabilität von Partnerschaften}

$\mathrm{Zu}$ Auswirkungen von Beschäftigungsbedingungen auf die Stabilität von Partnerschaften liegen bisher nur rudimentäre Ergebnisse vor. Falls Leiharbeit und unsichere Beschäftigung das Trennungsrisiko erhöhen sollten, sind die Auswirkungen jedenfalls eher gering, während lange Pendelstrecken das Risiko signifikant erhöhen. Bisherigen Untersuchungen zufolge sind belastende Arbeitsbedingungen eher für die Qualität der Partnerschaft und Partnerschaftskonflikte als für das Trennungsrisiko bedeutsam (Diewald et al. 2013).

\subsubsection{Freizeit}

Freizeit ist die Zeit, die der Erholung von den Arbeitsanforderungen in Beruf und Haushalt dient. Sie ist notwendig, um sich von Arbeitsbelastungen erholen zu können. Diese kann passive Erholung oder auch Aktivitäten in anderen sozialen Zusammenhängen wie der sozialen Partizipation in Netzwerken und Vereinen. Für Erholung ebenso wie für eine gesunde Lebensführung zur Krankheitsprävention sind insbesondere soziale Aktivitäten in der Freizeit und physische Aktivität wichtig (Sonnentag und Zijlstra 2006). Der Bedarf daran steigt mit hohen Arbeitsanforderungen, allerdings führen körperlich anspruchsvolle Tätigkeiten und häufige Überstunden im Gegenteil oft zu geringerer physischer Aktivität in der Freizeit (Schneider und Becker 2005).

Insgesamt sind Auswirkungen von Beschäftigungsbedingungen auf die Freizeit geringer als beim Familienleben (zusammenfassend: Sonnentag und Fritz 2010). Vergleichbar zur Forschung zu wahrgenommenen Konflikten zwischen Berufs- und Privatleben hat sich auch in der Freizeitforschung gezeigt, dass eine nicht in der Verfügung der Arbeitnehmer liegende Flexibilisierung von Arbeitszeiten und Arbeitsorten zu Koordinationsproblemen von Freizeitaktivitäten insbesondere dann führt, wenn es sich um mit anderen verbrachte Zeit und regelmäßige Aktivitäten etwa im Sport und generell in Vereinen handelt (Hallberg 2003). Cornwell und Warburton (2014) stellen bezüglich Schichtarbeit hierzu fest, dass eine Spätschicht die Teilnahme an formellen Aktivitäten wie Freiwilligenarbeit oder Engagement in der Kirchengemeinde reduziert, während Nachtschichtarbeitende ihren Zeitplan um die formellen Aktivitäten herum planen können, aber dafür reduzierte informelle Aktivitäten aufweisen. Schichtarbeit während der Woche wirkt sich nicht negativ auf das Engagement am Wochenende aus.

Die meisten Akteure versuchen Arbeit und Freizeit so zu koordinieren, dass sie möglichst viel Freizeit gemeinsam mit Familie und Freunden verbringen können (Carriero et al. 2009). Speziell am Wochenende sind davon gemeinsame Familienaktivitäten betroffen (Brown et al. 2011). Am Beispiel holländischer dual-earner couples mit Kindern konnte allerdings auch eine alternative Strategie nachgewiesen werden: Hier versuchten Partner ihre Arbeitszeiten so zu koordinieren, dass sich ihre Arbeitszeiten möglichst nicht überschneiden, damit wenigstens immer ein Partner 
Zeit mit den Kindern verbringen konnte (Carriero et al. 2009; van Klaveren und van den Brink 2007). Laut Lesnard (2008) ist diese Strategie weniger auf den freien Willen und die Entscheidung der Partner zurückzuführen, sondern vielmehr eine nicht intendierte Folge der von Unternehmen vorgegebenen Arbeitspläne. Lesnard (2008) findet diese Konstellation deshalb besonders häufig bei Berufsgruppen mit niedriger Qualifikation am unteren Ende der sozialen Leiter vor.

\section{Digitalisierung und Beschäftigungsmerkmale: Was ändert sich für wen?}

Die Digitalisierung verändert Arbeit entlang einer Reihe von Arbeitsplatz- und Tätigkeitsmerkmalen (siehe Mlekus, Ötting \& Maier in diesem Band), wie sie auch vorher schon im Hinblick auf Auswirkungen auf das Privatleben thematisiert und untersucht wurden. Insofern diese mit zunehmender Digitalisierung von Arbeitsplätzen zunehmen, kann von diesen Untersuchungen auf potenzielle Auswirkungen von Digitalisierung auch für die Gegenwart und Zukunft geschlossen werden.

Im Folgenden werden zunächst Beschäftigungsmerkmale beschrieben, die durch die Einführung cyber-physikalischer Systeme (potenziell) beeinflusst werden können und auf die private Lebensführung von Arbeiternehmern und Arbeitnehmerinnen verschiedener Beschäftigtengruppen wirken.

\subsection{Arbeitsaufgaben und Arbeitsbelastung}

Durch die Digitalisierung von Arbeit werden repetitive Tätigkeiten weniger häufig von Menschen, sondern zunehmend von Maschinen ausgeführt, da sog. RoutineTätigkeiten programmierbar auf diese übertragen werden können (Frey und Osborne 2013; für Deutschland Bonin et al. 2015; kritisch dazu Pfeiffer und Suphan 2015). Eine Studie des Bundesministeriums für Arbeit und Soziales zeigt, dass hinsichtlich der Anforderungen (teilweise) digitalisierter Arbeit für die verbliebenen Arbeitsplätze eher Belastungssteigerungen als Arbeitserleichterungen wahrgenommen werden (BMAS Monitor 2016c). Dies ist darauf zurückzuführen, dass für Beschäftigte mit einem niedrigen Grad formaler Ausbildung und Qualifikation die Gefahr besteht, dass einfachere Aufgaben substituiert und die verbliebenen Aufgaben, etwa das Fügen und Prüfen in der automatisierten Produktion, erhebliche Konzentration und Fingerfertigkeit erfordern. Allerdings kann dies auch bei hoch qualifizierten Tätigkeiten zu einer Komplexitätssteigerung führen. Nicht-Routine-Tätigkeiten, wie es sie zum Beispiel in Berufsfeldern mittleren und vor allem hohen Qualifikationsniveaus gibt, können derzeit nicht vollkommen durch Assistenz- und Softwaresysteme übernommen, jedoch aber unterstützt werden (Wolter et al. 2015). In der Folge kann auch dort der Wegfall von Routinetätigkeiten die an sich wünschenswerte Komplexität und Vielfalt von Arbeit zu sehr verdichten.

Zahlreiche Tätigkeiten werden zudem abstrakter, da Arbeitshandlungen, -objekte und -prozesse durch Digitalisierung in abstrahierte und textuelle Information über- 
setzt werden (,the electronic text“; Zuboff 1988). Auch der Grad der Vernetzung mit anderen Arbeitsplätzen steigt. Zugleich sinkt der Grad der Nachvollziehbarkeit von Arbeitsprozessen für einen Großteil der Beschäftigtengruppen. In digitalisierten Arbeitswelten kann so das Paradox der Automation (,,ironies of automation“; Bainbridge 1983) sichtbar werden, wonach Beschäftigten das Improvisieren in ungeplanten Situationen umso schwieriger erscheint, je stärker das System, das sie für den Fall einer Störung überwachen, automatisiert ist (Hirsch-Kreinsen 2014). Daraus kann eine geringe Nachvollziehbarkeit von Arbeitsprozessen und zunehmende Entfremdung resultieren und in Form erhöhten Arbeitsdrucks oder psychischer Belastung Einfluss auf die Qualität privater Zeit haben.

\subsection{Beschäftigungsunsicherheit}

Bekannte Formen unsicherer Beschäftigung in Form befristeter Arbeitsverträge und prekärer Beschäftigung wie geringfügige Beschäftigung oder Leiharbeit werden derzeit ergänzt durch die Vergabe einzelner Arbeitsaufgaben über elektronische Plattformen (Leimeister und Zogaj 2013). Unter einem solchen „Crowdsourcing“ bzw. „Crowdworking“"versteht man vernetztes Arbeiten, bei dem i. d. R. Internetnutzer und -nutzerinnen bestimmte Aufgaben und Projekte für Unternehmen bearbeiten, ohne fest angestellt zu sein. Crowdworking wird derzeit vor allem von Unternehmen in der Informationswirtschaft genutzt, jedoch zeichnet sich auch ein großes Potenzial im verarbeitenden Gewerbe ab. Insofern dadurch reguläre Beschäftigung substituiert wird, nimmt unsichere Beschäftigung in Form von Solo-Selbstständigkeit zu (Albrecht und Ammermüller 2016).

Die Wahrnehmung eines steigenden Substitutionspotenzials durch Automatisierung von auch komplexen, kognitiven Tätigkeiten zeigt sich auch als subjektive Wahrnehmung in Beschäftigtenbefragungen: Daran, in den nächsten zehn Jahren aufgrund der Digitalisierung von Arbeit durch Maschinen ersetzt zu werden, glauben immerhin $17 \%$ der Mittel- und $9 \%$ der Mittelhochqualifizierten, insgesamt sind es $13 \%$ der Beschäftigten (BMAS Monitor 2016c). In einer anderen Studie (EU Kommission 2015) gaben $26 \%$ der befragten abhängig Beschäftigten an, dass ihre Arbeitstätigkeit in Zukunft zumindest teilweise durch „Roboter “ ausgeführt werden könnte.

\subsection{Räumliche Verteilung der Arbeit}

Kabellose Technologien (zum Beispiel Cloud-Speicher, mobiles Internet, Tablets und Smartphones) ermöglichen zunehmend ortsungebundenes Arbeiten vor allem im Dienstleistungsbereich. Die damit verbundenen Probleme, aber auch Möglichkeiten einer Entgrenzung von Berufs- und Privatleben werden damit zunehmen. In welche Richtung das Pendel ausschlägt, steht nicht von vorneherein fest, sondern ist entscheidend von innerbetrieblichen Gestaltungspraxen von Konzepten wie dem „Home Office“ abhängig (BMAS Monitor Mobiles und entgrenztes Arbeiten 2015). 


\subsection{Arbeitszeitumfang und -flexibilität}

Es gibt keine klare Prognose, ob in digitalisierten Arbeitsumgebungen der Umfang der zu leistenden Arbeitszeit reduziert oder gesteigert ist. Jedoch wird die Lage von Arbeitszeit jenseits klar abgegrenzter Arbeitszeiten flexibilisiert und in Richtung ständiger Erreichbarkeit verschoben. Allerdings erlaubt der derzeitige Stand der Forschung noch keine Prognose, inwiefern digitalisierte Arbeitsumgebungen eher eine rein durch Rationalisierungsüberlegungen induzierte, für die Beschäftigten tendenziell ,rücksichtslose“ Flexibilisierung mit sich bringen oder aber mehr selbstbestimmte Flexibilität zulassen, so dass Mitarbeiter Unternehmensanforderungen mit ihren eigenen Bedürfnissen in Einklang bringen können (für letztere Variante siehe Bauer et al. 2014). Die mit Digitalisierung einhergehenden größeren Möglichkeiten einer Permeabilität räumlicher und zeitlicher Grenzen zwischen Arbeit und privater Lebensführung in flexiblen Arbeitssettings können je nach persönlicher Lebenssituation als vor- oder nachteilig empfunden werden, unter Umständen können dabei die Bedürfnisse beider Seiten gleichermaßen zu ihrem Recht kommen (Mikfeld 2016). Zu Skepsis gibt eine Unternehmensbefragung der Wirtschaftsprüfungsgesellschaft Ernst \& Young (2016) Anlass, die hinsichtlich einer für die Beschäftigten überwiegend selbstbestimmten Flexibilisierung zeigt, dass Unternehmen weitgehend diffuse Vorstellungen über die Effekte von digitalisierter Wertschöpfung haben. „Erstaunliche Einigkeit“ bestehe jedoch darüber, dass der große Vorteil der Digitalisierung in der Möglichkeit liege, die Flexibilität der Produktion zu erhöhen und die Reaktionszeit des Unternehmens auf Kunden- oder Marktanforderungen zu senken. Insgesamt scheint deshalb eine Prognose positiver versus negativer Auswirkungen auf das Privatleben derzeit nicht möglich.

\subsection{Autonomie und Kontrolle}

Beschäftigte nehmen strukturellen Wandel wie die Digitalisierung von Arbeit wahr, interpretieren diesen und handeln ihrer Einschätzung und den eigenen Ressourcen entsprechend: „Es findet eine reflexive Praxis statt, in der Mitarbeiter sich die eigene Arbeitssituation quasi ,aneignen“, spezifische Prioritäten entwickeln und eigene Grenzen ziehen." (Lohr 2013). Auch für den Umgang mit Digitalisierung wird gerade für Deutschland mit seiner breiten berufsfachlichen Qualifizierung angenommen, dass die Voraussetzungen dafür vergleichsweise günstig sind (Pfeiffer und Suphan 2015). Durch Digitalisierung beförderte Möglichkeiten flexibler Arbeitsfreiräume werden individuell als Autonomie gedeutet und zugleich unternehmerisch in Anforderungen übersetzt. Anforderungen können dabei sowohl vom Unternehmen an Beschäftigte gestellt werden, als auch Anforderungen von Beschäftigten an sich selbst bzw. die eigene Arbeitsleistung sein. Wie schon bei der Flexibilisierung der Arbeitszeit scheint es derzeit unmöglich zu entscheiden, ob sich dies primär eher als Gewinn an Gestaltungsautonomie oder als schwierige Anforderung an Selbstregulation niederschlägt. Dies wird in der Befragung des BMAS „Monitor Digitalisierung“ deutlich: $32 \%$ der befragten Beschäftigten nehmen eine erhöhte Arbeits- 
platzautonomie wahr, aber gleichzeitig erfahren $56 \%$ eine erhöhte Anforderung an Arbeitsleistung (BMAS Monitor 2016c). Arbeitsdruck, extrinsischen oder intrinsischen Ursprungs, erhöht als nervliche Belastung Konflikte zwischen Arbeit und privater Lebensführung. Das heißt, in Einklang mit früheren Untersuchungen scheinen auch die mit Digitalisierung verknüpften Komplexitäts-, Abstraktions-, Kommunikations- und Problemlösungsanforderungen ambivalent im Hinblick auf Auswirkungen auf das Privatleben: Unter dem Strich können die damit verbundenen Belastungen sich sowohl negativ auswirken als auch durch die gewonnenen Handlungsspielräume Vereinbarkeitschancen erhöhen.

Adaptive Mensch-Maschine-Schnittstellen bzw. CPS benötigen Zugriff auf unterschiedliche Daten zum Zustand eines Beschäftigten, um sich auf ihn einzustellen (Hörmann und Rückert 2017). Daraus resultiert eine neue Qualität von Überwachungsmöglichkeiten (Krzywdzinski et al. 2015). Neben Überwachungsinstrumenten herkömmlicher Leistungskontrolle wie visueller Monitorisierung, Messung der Leistungsgeschwindigkeit, Fehlerraten und Qualitätsmarker, stehen durch Aufmerksamkeitsüberwachungssysteme, die Messung von Bio-Daten und Standortbestimmungstechnologien (GPS, RFID) Instrumente neuer Art zur Überwachung und Kontrolle von Beschäftigten zu Verfügung. Ortmann (2014) zeigt die besonderen Anforderungen, die der Einsatz dieser Technologie beinhaltet am Beispiel von Beschäftigten eines Textillagers und einer Bibliothek auf: Durch das Komprimieren und Digitalisieren von Informationen in Form von Mikro-Chips und Transpondern, die in Objekte integriert werden (z. B. bei EY 2016), lässt sich die Nach- und Rückverfolgbarkeit zahlreicher Schritte des Produktionsprozesses maximieren. Dies betrifft vor allem die sowieso schon eher unter Kontrolle stehenden Beschäftigten in einfacheren Berufen, ist jedoch nicht darauf beschränkt. Entgegen der in den „Umsetzungsempfehlungen für Industrie 4.0“ (Kagermann et al. 2013) vorgestellten Vision dürfte dadurch die sog. Work-Life-Balance eher erschwert als erleichtert werden.

\section{$4 \quad$ Neue Aspekte digitalisierter Arbeit und vorliegende Untersuchungen zum Zusammenhang zwischen digitalisierter Arbeit und Privatleben}

Nachfolgend werden schlaglichtartig weitgehend neue Aspekte der Arbeitsgestaltung in digitalisierten Arbeitswelten vorgestellt. Diese beziehen sich im Kern auf neue Formen der Interaktion zwischen Mensch und Maschine, welche sich in ihrer Qualität von bisheriger unterscheidet und sowohl in das Arbeitsleben als auch die private Lebensführung reicht.

$\mathrm{Da}$ aussagekräftige, empirische Studien zum Zusammenhang zwischen einer Digitalisierung der Arbeit und privater Lebenswelt noch ausstehen, spiegelt sich der gegenwärtige Stand der Forschung in der Hauptsache in Experteninterviews, Unternehmensbefragungen und Expertisen in Form von allgemeinen Trendaussagen. Diese Expertisen und Unternehmensbefragungen werden abschließend von uns eingeordnet. 


\subsection{Algorithmen als Entscheidungsinstanz}

Zukünftig werden Entscheidungen nicht mehr allein von menschlichen Entscheidungsinstanzen, zum Beispiel Führungskräften, getroffen, sondern vermehrt durch autonome technische Systeme. Beim derzeitigen Stand der Forschung ist noch ungeklärt, ob sich dadurch die Chance von Beschäftigten reduziert, Arbeitsprozesse und -entscheidungen nachvollziehen zu können oder ob Entscheidungen technischer Systeme unter spezifischen Voraussetzungen (bspw. wenn dadurch die Leistung eines Teams verbessert wird, Gombolay und Shah 2014) begrüßt werden.

Gerade für diese Art von Entscheidungen wird es wichtig werden, sie so zu gestalten, dass sie auch als fair wahrgenommen werden (Töniges et al. 2017; Engels et al. im Druck).

Wesentlich für algorithmenbasierte Entscheidungen ist die Vermeidung diskriminierender Algorithmen bezüglich bestimmter Personengruppen, wie sie zum Beispiel bei Einreisekontrollen oder Kreditvergabeentscheidungen bereits bekannt sind. Diskriminierende Effekte zeigen sich, wenn die einem System zugrunde liegenden Algorithmen auf Daten basieren, die bereits strukturelle Diskriminierung aufweisen (Dewes 2015). Algorithmen können jedoch durchaus umgekehrt Stereotypen konterkarieren und insbesondere unbewusste Diskriminierungen vermeiden helfen.

\subsection{Neue Formen der Mensch-Maschine-Interaktion/- Kommunikation}

Spezifische neue Merkmale digitalisierter Arbeit sehen wir in neuen Formen bzw. einer erhöhten Intensität der Mensch-Maschine-Interaktion und zahlreichen Varianten der Interface-Kommunikation. Die Verstärkung dieser Formen der InterfaceKommunikation mündet in eine neue Qualität mittelbarer Kommunikation. Der Einfluss solcher mittelbarer Kommunikation auf die Qualität sozialer Einbindungen, insbesondere durch sogenannte ,neue Medien“, ist bisher eher für den Bereich der privaten Lebensführung untersucht worden. Dort sind die Auswirkungen gering. Entgegen kulturpessimistischer Annahmen (Kraut et al. 1998; Putnam 1995, 2000; Rice 2002) zum Einfluss sozialer Medien auf Kommunikation und die Qualität sozialer Netzwerke bleibt sowohl die Größe der Netzwerke als auch die Beziehungsqualität mit Freunden, die offline getroffen werden, und innerhalb der Familie weitgehend unverändert (Shklovski et al. 2006; Vergeer und Pelzer 2009). Die Überschneidung von online- und offline-Netzwerken ist relativ groß (Subrahmanyam et al. 2008). Soziale Medien werden vor allem dafür benutzt, soziale Kontakte aufrechtzuerhalten, wohingegen das Schließen neuer Kontakte eher die Ausnahme ist (Ellison et al. 2011; Reich et al. 2012; Subrahmanyam et al. 2008).

Allerdings scheint die digitale Anbindung an die Arbeit rund um die Uhr das Abschalten in Familie und Freizeit zu beeinträchtigen. Dazu trägt auch bei, dass Unberechenbarkeit und ein Autonomieverlust dadurch entstehen, dass im Voraus unklar ist, wann und von wem man kontaktiert und zu Reaktionen herausgefordert wird. Allerdings spielen auch hier wieder persönliche Lebensumstände und Organi- 
sationspraktiken eine große moderierende Rolle, so dass es wohl keine uniformen Auswirkungen auf das Privatleben gibt bzw. geben wird (Derks et al. 2014; Demerouti et al. 2014).

\subsection{Konkurrenz in Mensch-Maschine-Interaktion}

Inwieweit dies auch für weiter entwickelte digitale Dienstleistungen mit dem Anspruch gilt, grundlegende, emotionale Bedürfnisse (wie zum Beispiel emotionale Nähe, körperliche Intimität oder Aufmerksamkeit) zunehmend befriedigen zu können, ist derzeit nicht abzusehen - und nicht auszuschließen. Im Bereich der Aufmerksamkeitsökonomie stehen menschliche Akteure und Akteurinnen zunehmend in Konkurrenz zu maschinell erstellten Inhalten. ${ }^{1}$ Digitale Formen der Zuwendung erscheinen durch ihre konstante Verfügbarkeit im Vergleich zu den reziproken Prozessen zwischenmenschlicher Kontakte, deren Optimierung und Personalisierung zudem kaum in dem Maße möglich ist wie durch Algorithmen, quantitativ und qualitativ überlegen. Visionen einer solchen emotionalen Überlegenheit humanoider Systeme gegenüber menschlichen sozialen Fähigkeiten bietet Hollywood bereits in Filmen wie „Ex-Machina“ (2015) und „her“ (2013) an.

\subsection{Vorliegende Untersuchungen zum Zusammenhang zwischen digitalisierter Arbeit und Privatleben: Expertisen und Unternehmensbefragungen}

Unternehmens- und Expertenbefragungen zu Stand und Perspektiven der Digitalisierung stellen keine aussagekräftigen Untersuchungen dar, doch können sie widerspiegeln, inwiefern potenzielle Auswirkungen auf das Privatleben überhaupt thematisiert werden, und ob dabei eher Hoffnungen oder Befürchtungen eine Rolle spielen. Einen umfassenden Überblick solcher Expertisen bieten Ittermann et al. (2015). Es zeigt sich, dass Auswirkungen der Digitalisierung auf die private Lebensführung quasi nie thematisiert werden. Die „Umsetzungsempfehlungen für das Zukunftsprojekt Industrie 4.0“ (Kagermann et al. 2013) stellen Visionen und Handlungsempfehlungen dar, die weitgehend auf Expert/innen-Meinungen von Unternehmens- und Branchenvertreter/innen und der Autoren selbst basieren. Private Lebensführung in digitalisierten Arbeitswelten findet hier nur am Rande in den Umsetzungsempfehlungen als „Work-Life-Balance“ Erwähnung, ohne dass geklärt würde, wo und warum digitalisierte Arbeitsumgebungen eine besondere Herausforderung hierfür darstellen würden.

Expliziter wird das Thema Work-Life-Balance in der Studie „Wertewelten Arbeit 4.0“ (2016) angesprochen, die im Auftrag des Bundesministeriums für Arbeit und

\footnotetext{
${ }^{1}$ Zum Beispiel durch das rtr text Programm, durch welches Sportberichterstattung automatisiert wird (http://www.retresco.de/rtr_textengine. Zugegriffen am 01.04.2016).
} 
Soziales veröffentlicht wurde. Dabei wurden 1200 Personen zu Vorstellungen zu Arbeit in gegenwärtigen, früheren und künftigen Jahrzehnten befragt. Die dabei gebildeten Wertewelten erheben den Anspruch, Ideale, Hoffnungen und Befürchtungen zum Thema Arbeiten abzubilden. Einige der vorgestellten Wertewelten enthalten Vorstellungen zu Aspekten der Synchronisation und Verwirklichungsmöglichkeiten privater Lebensziele in Arbeit (BMAS Wertewelten 2016e). Die WerteweltenPerspektive verweist damit implizit auf die Abhängigkeit der Wahrnehmung von Digitalisierungsprozessen von persönlichen Lebenszielen, was eine grundlegende Perspektive der Digitalisierungsdebatte sein sollte. Insgesamt sind Befürchtungen hinsichtlich negativer Auswirkungen auf das Privatleben eher gering ausgeprägt.

\section{$5 \quad$ Fazit}

Insgesamt steht der möglichen Relevanz von Digitalisierungsprozessen in der Arbeitswelt für die Gestaltung des Privatlebens bisher eine äußerst magere Forschungslage gegenüber. Zudem zeigt der Überblick über die Beschäftigungsmerkmale, dass die Digitalisierung häufig wohl eher bereits längere Zeit bestehende Tendenzen - vor allem räumliche und zeitliche Flexibilisierung und Beschäftigungsunsicherheit - weiter vorantreibt als völlig neue Tendenzen generiert (BMAS Foresight Studie 2016a). Aufgrund des noch sehr geringen Umfangs methodisch anspruchsvollerer empirischer Studien sind wir allerdings bisher weitgehend auf indirekte Schlussfolgerungen aus früheren Studien zu Beschäftigungsmerkmalen angewiesen, die sich, noch ohne den derzeitigen Stand der Digitalisierung, als einflussreich für verschiedene Aspekte der Gestaltung des Privatlebens erwiesen haben. Hierbei haben wir so weit wie möglich berücksichtigt, dass ein und dieselben Digitalisierungsprozesse für verschiedene darin involvierte Beschäftigtengruppen gänzlich unterschiedliche Konsequenzen haben können.

Auch angesichts der Heterogenität und Komplexität der unter dem Stichwort Digitalisierung subsumierten Prozesse lassen sich die bisherigen Evidenzen kaum zu einer Gesamteinschätzung zusammenfassen. Am wahrscheinlichsten scheint uns, dass zunehmende Click-/Cloud-/Crowdworking Auswirkungen auf das Privatleben haben können. Zum einen könnten die damit einhergehenden Beschäftigungsunsicherheiten das Eingehen stabiler, verpflichtungsreicher Beziehungen verzögern. Zum anderen werden damit aber auch Gestaltungsfreiräume wachsen, die eine den eigenen Bedürfnissen folgende Freizeit- und Beziehungsgestaltung erleichtern. Damit ist diese anwachsende Gruppe auch ein Beispiel für eine bemerkenswerte Übereinstimmung zwischen vielen älteren und neuen, direkt auf Digitalisierung bezogenen Untersuchungen: Es dürfte wohl gar keinen uniformen Effekt verschiedener Digitalisierungsprozesse auf verschiedene Beschäftigtengruppen mit unterschiedlichen beruflichen und privaten Lebensverhältnissen geben. Vielmehr dürften sich im Zusammenspiel mit diesen Lebensverhältnissen sehr unterschiedliche Konsequenzen ergeben. Diese wiederum dürften in erheblichem Umfang von der konkreten Ausgestaltung und begleitenden Regelung der Digitalisierung durch rechtli- 
che Rahmenbedingungen, industrielle Beziehungen und organisationale Praktiken abhängig sein.

\section{Literatur}

Adkins, C. L., \& Premeaux, S. F. (2012). Spending time: The impact of hours worked on work-family conflict. Journal of Vocational Behavior, 80(2), 380-389.

Albrecht, T., \& Ammermüller, A. (2016). Kein Ende der Arbeit in Sicht. In BMAS 2016 Werkheft 1: Digitalisierung der Arbeitswelt (S. 40-46). Berlin: Bonifatius GmbH.

Allen, T. D. (2001). Family-supportive work environments: The role of organizational perceptions. Journal of Vocational Behavior, 58(3), 414-435.

Allen, T. D., Herst, D. E. L., Bruck, C. S., \& Sutton, M. (2000). Consequences associated with work-to-family conflict: A review and agenda for future research. Journal of Occupational Health Psychology, 5(2), 278-308.

Bainbridge, L. (1983). Ironies of automation. Automatica, 19, 775-780.

Bakker, A. B., \& Demerouti, E. (2007). The Job Demands-Resources model: State of the art. Journal of Managerial Psychology, 22(3), 309-328.

Bauer, W., Schlund, S., \& Marrenbach, D. (2014). Industrie 4.0: Volkswirtschaftliches Potenzial für Deutschland. Studie. Berlin/Stuttgart. https://www.bitkom.org/Publikationen/2014/Studien/Stu die-Industrie-4-0-Volkswirtschaftliches-Potenzial-fuer-Deutschland/Studie_Industrie_40.pdf. Zugegriffen am 17.11.2015.

Beham, B., \& Drobnič, S. (2010). Satisfaction with work-family balance among German office workers. Journal of Managerial Psychology, 25(6), 669-689.

BMAS. (2015). Monitor Digitalisierung am Arbeitsplatz. Aktuelle Ergebnisse einer Betriebs- und Beschäftigtenbefragung. http://www.bmas.de/DE/Service/Medien/Publikationen/a875-monitordigitalisierung-am-arbeitsplatz.html. Zugegriffen am 30.04.2016.

BMAS. (2016a). Foresight-Studie Digitale Arbeitswelt. http://www.bmas.de/SharedDocs/Down loads/DE/PDF-Publikationen/Forschungsberichte/f463-digitale-arbeitswelt.pdf?_blob=publicati onFile\&v $=2$. Zugegriffen am 30.04.2016.

BMAS. (2016b). Grünbuch Arbeiten 4.0. http://www.bmas.de/SharedDocs/Downloads/DE/PDFPublikationen-DinA4/gruenbuch-arbeiten-vier-null.pdf? blob=publicationFile. Zugegriffen am 30.04.2016.

BMAS. (2016c). Monitor Mobiles und entgrenztes Arbeiten: Aktuelle Ergebnisse einer Betriebsund Beschäftigtenbefragung. http://www.bmas.de/SharedDocs/Downloads/DE/PDF-Publikatio nen/a873.pdf? blob=publicationFile\&v=2. Zugegriffen am 30.04.2016.

BMAS. (2016e). Wertewelten Arbeit 4.0. https://www.arbeitenviernull.de/mitmachen/wertewelten/ studie-wertewelten.html. Zugegriffen am 30.04.2016.

BMBF. (2015). Industrie 4.0. Innovationen für die Produktion von morgen (2. Aufl.) (April 2015). https://www.bmbf.de/pub/Industrie_4.0.pdf. Zugegriffen am 20.02.2017.

Böhm, S., \& Diewald, M. (2012). Auswirkungen belastender Arbeitsbedingungen auf die Qualität privater Lebensverhältnisse. WSI-Mitteilungen, 22, 103-112.

Bonin, H., Gregory, T., \& Zieran, U. (2015). Übertragung der Studie von Frey/Osborne (2013) auf Deutschland: Kurzexpertise im Auftrag des Bundesministeriums für Arbeit und Soziales. Zugegriffen am 17.11.2015.

Brown, K., Bradley, L., Lingard, H., Townsend, K., \& Ling, S. (2011). Labouring for leisure? Achieving work-life balance through compressed working weeks. Annals of Leisure Research, 14(1), 43-59.

Carlson, D. S., Kacmar, K. M., \& Williams, L. J. (2000). Construction and initial validation of a multidimensional measure of work-family conflict. Journal of Vocational Behavior, 56(2), 249-276. 
Carriero, R., Ghysels, J., \& van Klaveren, C. (2009). Do parents coordinate their work schedules? A comparison of Dutch, Flemish, and Italian dual-earner households. European Sociological Review, 25(5), 603-617.

Cooke, L. P., \& Gash, V. (2010). Wives' part-time employment and marital stability in Great Britain, West Germany and the United States. Sociology, 44(6), 1091-1108.

Cornwell, B., \& Warburton, E. (2014). Work schedules and community ties. Work and Occupations, 41(2), 139-174.

Demerouti, E., Derks, D., Lieke, L., \& Bakker, A. B. (2014). New ways of working. Impact on working conditions, work-family balance, and well-being. In C. Korunka \& P. Hoonakker (Hrsg.), The impact of ICT on quality of working life (S. 123-141). Dordrecht: Springer.

Derks, D., ten Brummelhuis, L. L., Zecic, D., \& Bakker, A. B. (2014). Switching on and off. Does smartphone use obstruct the possibility to engage in recovery activities? European Journal of Work and Organizational Psychology, 23(1), 80-90.

Dewes, A. (2015). Say hi to your new boss: How algorithms might soon control our lives. https:// events.ccc.de/congress/2015/Fahrplan/events/7482.html. Zugegriffen am 18.10.2017.

Diewald, M. (2003). Kapital oder Kompensation? Erwerbsbiographien von Männern und die sozialen Beziehungen zu Verwandten und Freunden. Berliner Journal für Soziologie, 2003(2), 213-238.

Diewald, M., Böhm, S., Graf, T., \& Hoherz, S. (2013). Paarbeziehungen und Erwerbsarbeit. Gender Zeitschrift für Geschlecht, Kultur und Gesellschaft, Sonderheft, 2, 99-119.

Drobnič, S., \& Guillén Rodríguez, A. M. (2011). Tensions between work and home: Job quality and working conditions in the institutional contexts of Germany and Spain. Social Politics: International Studies in Gender, State and Society, 18(2), 232-268.

Eichhorst, W., Kendzia, M. J., Schneider, H., \& Buhlmann, F. (2013). Neue Anforderungen durch den Wandel der Arbeitswelt. Kurzexpertise für die Enquete-Kommission ,Wachstum, Wohlstand, Lebensqualität" des Deutschen Bundestages (IZA Research Report No. 51) Bonn.

Ellison, N. B., Steinfield, C., \& Lampe, C. (2011). Connection strategies: Social capital implications of Facebook-enabled communication practices. New Media \& Society, 13(6), 873-892.

Engels, G., Maier, G. W., Ötting, S., Steffen, E., \& Teetz, A. (im Druck 2018). Gerechtigkeit in flexiblen Arbeits- und Managementprozessen. In S. Wischmann (Hrsg.), Zukunft der Arbeit: Eine praxisnahe Betrachtung. Berlin: Springer.

Europäische Kommission. (2015). Special Eurobarometer 427. Autonomous systems. Report. http://ec.europa.eu/public_opinion/archives/ebs/ebs_427_en.pdf. Zugegriffen am 29.04.2016.

EY (Ernst \& Young), \& Bitkom. (2016). Industrie 4.0: das unbekannte Wesen. http://www.bitkomresearch.de/Industrie-40. Zugegriffen am 18.10.2017.

Frey, C. B., \& Osborne, M. A. (2013). The future of employment: How susceptible are jobs to computerisation? http://www.oxfordmartin.ox.ac.uk/downloads/academic/The_Future_of_ Employment.pdf. Zugegriffen am 18.10.2017.

Gallie, D., \& Russell, H. (2009). Work-family conflict and working conditions in Western Europe. Social Indicators Research, 93(3), 445-467.

Goh, Z., Ilies, R., \& Schwind Wilson, K. (2015). Supportive supervisors improve employees' daily lives: The role supervisors play in the impact of daily workload on life satisfaction via work-family conflict. Journal of Vocational Behavior, 89, 65-73.

Gombolay, M. C., \& Shah, J. A. (2014). Challenges in collaborative scheduling of human-robot teams. Artificial intelligence for human-robot interaction: Papers from the 2014 AAAI fall symposium.

Gortz, A. (1973). Wege ins Paradies. Thesen zur Krise, Automation und Zukunft der Arbeit. Berlin: Rotbuch Verlag.

Greenhaus, J. H., \& Beutell, N. J. (1985). Sources of conflict between work and family roles. The Academy of Management Review, 10(1), 76-88.

Grzywacz, J. G., \& Butler, A. B. (2005). The impact of job characteristics on work-to-family facilitation: Testing a theory and distinguishing a construct. Journal of Occupational Health Psychology, 10(2), 97-109. 
Hallberg, D. (2003). Synchronous leisure, jointness and household labor supply. Labour Economics, 10, 185-203.

Hammer, L. B., Kossek, E. E., Yragui, N. L., Bodner, T. E., \& Hanson, G. C. (2009). Development and validation of a multidimensional measure of family supportive supervisor behaviors (FSSB). Journal of Management, 35(4), 837-856.

Hermann, M., Pentek, T., \& Otto, B. (2015). Design principles for Industrie 4.0 scenarios: A literature review (Working Paper Nr. 01/2015).

Hill, E. J., Erickson, J. J., Holmes, E. K., \& Ferris, M. (2010). Workplace flexibility, work hours, and work-life conflict: Finding an extra day or two. Journal of Family Psychology, 24(3), 349-358.

Hirsch-Kreinsen, H. (2014). Wandel von Produktionsarbeit: „Industrie 4.0“. WSI Mitteilungen, (06), 421-429. http://www.wiso.tu-dortmund.de/wiso/is/Medienpool/de/meldungen/Aufsatzzu-Industrie-4_0-erschienen/Hirsch-Kreinsen-Wandel-von-Produktionsarbeit-Industrie-4_0. pdf. Zugegriffen am 19.02.2015.

Hobson, B. M. (2014). Worklife balance: The agency \& capabilities gap. Oxford: Oxford University Press.

Hobson, B., \& Fahlén, S. (2009). Competing scenarios for European fathers: Applying Sen's capabilities and agency framework to work-family balance. The Annals of the American Academy of Political and Social Science, 624(1), 214-233.

Hörmann, T., \& Rückert, U. (2017). Vernetzte Arbeitsumgebungen: Körpernahe und tragbare Sensorik in der Arbeitswelt. In G. W. Maier, G. Engels \& E. Steffen (Hrsg.), Handbuch Gestaltung digitaler und vernetzter Arbeitswelten. Heidelberg: Springer.

Institut für Demoskopie Allensbach. (2014). Die Zukunft der digitalen Gesellschaft. Ergebnisse einer repräsentativen Bevölkerungsumfrage. http://www.digital-ist.de/fileadmin/content/DieThemen/Umfrage/Ergebnisse_Umfrage_komplett.pdf. Zugegriffen am 29.04.2016.

Ittermann, P., Niehaus, J., \& Hirsch-Kreinsen, H. (2015). Arbeiten in der Industrie 4.0: Trendbestimmungen und arbeitspolitische Handlungsfelder. http://www.wiso.tu-dortmund.de/wiso/de/ forschung/gebiete/fp-hirschkreinsen/aktuelles/meldungsmedien/20150721-Ittermann-et-al2015-Arbeiten-in-der-Industrie-4-0-HBS.pdf. Zugegriffen am 29.04.2016.

Kagermann, H., Wahlster, W., \& Helbig, J. (2013). Umsetzungsempfehlungen für das Zukunftsprojekt Industrie 4.0: Abschlussbericht des Arbeitskreises Industrie 4.0. Berlin: Forschungsunion Wirtschaft und Wissenschaft. http://www.forschungsunion.de/pdf/industrie_4_0_umsetzungs empfehlungen.pdf. Zugegriffen am 18.10.2017.

Klaveren, van C., \& van den Brink, H. (2007). Intra-household work time synchronization. Social Indicators Research, 84(1), 39-52.

Kraut, R., Patterson, M., Lundmark, V., Kiesler, S., Mukophadhyay, T., \& Scherlis, W. (1998). Internet paradox: A social technology that reduces social involvement and psychological wellbeing? American Psychologist, 53, 1017-1031.

Kreyenfeld, M. (2010). Uncertainties in female employment careers and the postponement of parenthood in Germany. European Sociological Review, 26(3), 351-366.

Krzywdzinski, M., Jürgens, U., \& Pfeiffer, S. (2015). Die vierte Revolution: Wandel der Produktionsarbeit im Digitalisierungszeitalter. WZB Mitteilungen, (149), 6-9. https://www.wzb.eu/sites/ default/files/publikationen/wzb_mitteilungen/s6-9juergensua.pdf. Zugegriffen am 16.11.2015.

Kurz, K. (2005). Die Familiengründung von Männern im Partnerschaftskontext. Eine Längsschnittanalyse zur Wirkung von Arbeitsmarktunsicherheiten. In A. Tölke \& K. Hank (Hrsg.), Männer - Das , vernachlässigte' Geschlecht in der Familienforschung. Sonderheft 4 der Zeitschrift für Familienforschung (S. 178-197). Wiesbaden: VS Verlag für Sozialwissenschaften.

Laß, I. (2016). Waiting for the right moment? The effect of employment-related insecurity on first childbirth among couples in West Germany. Unveröff. Manuskript, Universität Bielefeld.

Leimeister, J. M., \& Zogaj, S. (2013). Neue Arbeitsorganisation durch Crowdsourcing. Eine Literaturstudie (Arbeitspapier Nr. 287). Düsseldorf: Hans Böckler Stiftung.

Lesnard, L. (2008). Off-scheduling within dual-earner couples: An unequal and negative externality for family time. American Journal of Sociology, 114(2), 447-490. 
Lohr, K. (2013). Subjektivierung von Arbeit. In H. Hirsch-Kreinsen \& H. Minssen (Hrsg.), Lexikon der Arbeits- und Industriesoziologie (LAIS) (S. 430-437). Berlin: Ed. Sigma.

Mikfeld, B. (2016). Trends, Diskurse, Klärungsbedarfe. In BMAS 2016 Werkheft 1: Digitalisierung der Arbeitswelt (S. 16-21). Berlin: Bonifatius GmbH.

Moen, P., Kelly, E. L., Fan, W., Lee, S.-R., Almeida, D., Kossek, E. E., \& Buxton, O. M. (2016). Does a flexibility/support organizational initiative improve high-tech employees' well-being? Evidence from the work, family, and health network. American Sociological Review, 81(1), $134-164$.

Ortmann, U. (2014). Der Leistungsanspruch von RFID: Mit Popitz durch die Informationsgesellschaft. Arbeits- und Industriesoziologische Studien (AIS), 7(1), 75-86. http://www.ais-studien.de/ uploads/tx_nfextarbsoznetzeitung/AIS-14-01-6Ortmannfinal.pdf. Zugegriffen am 18.10.2017.

Pausch, S., Reimann, M., Abendroth, A., \& Diewald, M. (2016). Work-life integration of dualearner couples. Spillover, crossover, and accumulation of workplace demands and resources within partnerships. Psychosociological Issues in Human Resource Management, 4(1), 70-95.

Pfeiffer, S., \& Suphan, A. (2015). Der AV-Index: Lebendiges Arbeitsvermögen und Erfahrung als Ressourcen auf dem Weg zu Industrie 4.0 (Working Paper der FG Soziologie No. 01/2015). Universität Hohenheim. http://www.sabine-pfeiffer.de/files/downloads/2015-Pfeiffer-Suphandraft.pdf. Zugegriffen am 16.11.2015.

Putnam, R. D. (1995). Tuning in, tuning out: The strange disappearance of social capital in America. Ps-Political Science \& Politics, 28(4), 664-683.

Putnam, R. D. (2000). Bowling alone: The collapse and revival of American community. New York: Simon \& Schuster.

Reich, S. M., Subrahmanyam, K., \& Espinoza, G. (2012). Friending, IMing, and hanging out faceto-face: Overlap in adolescents' online and offline social networks. Developmental Psychology, 48(2), 356-368.

Reimann, M. (2016). The moderating role of overcommitment in the relationship between psychological contract breach and employee mental health. Journal of Occupational Health, 58(4-5), 425-433.

Reimann, M., Diewald, M., \& Pausch, S. (2016, under review). Work-family conflict in Germany: Psychological contracts as part of employment relationships in work-family research. Journal of Vocational Behavior.

Rice, R. E. (2002). Primary issues in Internet use: Access, civic and community involvement, and social interaction and expression. In L. Lievrouw \& S. Livingstone (Hrsg.), Handbook of new media: Social shapings and consequences of ICTs (S. 105-129). London: Sage.

Rousseau, D. M. (1995). Psychological contracts in organizations. Understanding written and unwritten agreements. Thousand Oaks/London/New Delhi: Sage.

Schieman, S., Milkie, M. A., \& Glavin, P. (2009). When work interferes with life: Work-nonwork interference and the influence of work-related demands and resources. American Sociological Review, 74(6), 966-988.

Schier, M., \& Jurczyk, K. (2007). „Familie als Herstellungsleistung“ in Zeiten der Entgrenzung. Aus Politik und Zeitgeschichte, Nr. 34. Bundeszentrale für politische Bildung, 20.08.2007.

Schmitt, C. (2008). The effects of labour market participation on fertility decisions: Gender differences in cross-national perspective. Dissertation, Bielefeld.

Schmitt, C. (2012). Labour market integration, occupational uncertainty, and fertility choices in Germany and the UK. Demographic Research, 26, 253-292.

Schneider, S., \& Becker, S. (2005). Prevalence of physical activity among the working population and correlation with work-related factors: Results from the First German National Health Survey. Journal of Occupational Health, 47, 414-423.

Sennett, R. (1998). Der flexible Mensch. Die Kultur des neuen Kapitalismus. Berlin: Berlin-Verlag.

Shklovski, I., Kiesler, S., \& Kraut, R. (2006). The Internet and social interaction: A meta-analysis and critique of studies, 1995-2003. In R. Kraut, M. Brynin \& S. Kiesler (Hrsg.), Computers, phones, and the Internet: Domesticating information technology (Oxford series in humantechnology interaction, S. 251-264). New York: Oxford University Press. 
Sonnentag, S., \& Fritz, C. (2010). Arbeit und Privatleben: Das Verhältnis von Arbeit und Lebensbereichen außerhalb der Arbeit aus Sicht der Arbeitspsychologie. In N. Birbaumer, et al. (Hrsg.), Enzyklopädie der Psychologie (Bd. 1: Arbeitspsychologie, S. 669-704). Göttingen: Hogrefe.

Sonnentag, S., \& Zijlstra, F. R. H. (2006). Job characteristics and off-job activities as predictors of need for recovery, well-being, and fatigue. Journal of Applied Psychology, 91(2), 330-350.

Subrahmanyam, K., Reich, S. M., Waechter, N., \& Espinoza, G. (2008). Online and offline social networks: Use of social networking sites by emerging adults. Journal of Applied Developmental Psychology, 29(6), 420-433.

Töniges, T., Ötting, S., Wrede, B., Maier, G. W., \& Sagerer, G. (2017). An emerging decision authority. Adaptive cyber-physical system design for fair human machine interaction and decision processes. In H. Song, D. B. Rawat, S. Jeschke \& C. Brecher (Hrsg.), Intelligent data-centric systems. Sensor collected intelligence (S. 419-430). Amsterdam: Elsevier.

Vergeer, M., \& Pelzer, B. (2009). Consequences of media and Internet use for offline and online network capital and well-being. A causal model approach. Journal of Computer-Mediated Communication, 15(1), 189-210.

Voydanoff, P. (2004). The effects of work demands and resources on work-to-family conflict and facilitation. Journal of Marriage and Family, 66(2), 398-412.

Wolter, M. I., Mönnig, A., Hummel, M., Schneemann, C., Weber, E., Zika, G., et al. (2015). Industrie 4.0 und die Folgen für Arbeitsmarkt und Wirtschaft: Szenario-Rechnungen im Rahmen der BIBBIAB-Qualifikations- und Berufsfeldprojektionen (IAB-Forschungsbericht No. 08/2015). Institut für Arbeitsmarkt- und Berufsforschung. http://doku.iab.de/forschungsbericht/2015/fb0815.pdf. Zugegriffen am 15.11.2015.

Zuboff, S. (1988). In the age of the smart machine: The future of work and power. Oxford: Heinemann. 